\title{
Tailoring properties of natural deep eutectic solvents with water to facilitate their applications
}

\author{
Yuntao Dai ${ }^{\mathrm{a}}$, Geert-Jan Witkamp ${ }^{\mathrm{b}}$, Robert Verpoorte ${ }^{\mathrm{a}}$, Young Hae Choi ${ }^{\mathrm{a}, *}$ \\ ${ }^{a}$ Natural Products Laboratory, Institute of Biology, Leiden University, 2333 BE Leiden, The Netherlands \\ ${ }^{\mathrm{b}}$ Department of Biotechnology, Delft University of Technology, 2628 BC Delft, The Netherlands
}

\section{A R T I C L E I N F O}

\section{Article history:}

Received 12 October 2014

Received in revised form 8 February 2015

Accepted 30 March 2015

Available online 3 April 2015

\section{Keywords:}

Natural deep eutectic solvents

Stabilizing capacity

Quercetin

Carthamin

Water content

Hydrogen bonding

\begin{abstract}
A B S T R A C T
Previously it was demonstrated that natural deep eutectic solvents (NADES) are promising green solvents for the extraction of natural products. However, despite their potential, an obvious disadvantage of NADES is the high viscosity. Here we explored the dilution effect on the structures and physicochemical properties of NADES and their improvements of applications using quercetin and carthamin. The results of FT-IR and ${ }^{1} \mathrm{H}$ NMR experiments demonstrated that there are intensive $\mathrm{H}$-bonding interactions between the two components of NADES and dilution with water caused the interactions weaken gradually and even disappeared completely at around $50 \%(\mathrm{v} / \mathrm{v})$ water addition. A small amount of water could reduce the viscosity of NADES to the range of water and increase the conductivity by up to 100 times for some NADES. This study provides the basis for modulating NADES in a controllable way for their applications in food processing, enzyme reactions, pharmaceuticals and cosmetics.
\end{abstract}

(c) 2015 Elsevier Ltd. All rights reserved.

\section{Introduction}

A new type of green solvents, natural deep eutectic solvents (NADES) have been proposed by our group to extend the range of ionic liquids (ILs) and deep eutectic solvents (DES) and to explore their applications in life science fields (Choi et al., 2011; Dai, Spronsen, Witkamp, Verpoorte, \& Choi, 2013a; Dai, Verpoorte, \& Choi, 2014). Natural deep eutectic solvents are composed mostly of natural primary metabolites such as sugars, sugar alcohols, organic acids, amino acids, and amines and additionally often contain water in certain molar ratios. They are characterized by extensive intermolecular interactions (Choi et al., 2011; Dai et al., 2013a). A series of NADES possessing excellent properties as solvents has been developed. These include negligible volatility, liquid state even far below $0{ }^{\circ} \mathrm{C}$, a broad range of polarity, high solubilization power strength for a wide range of compounds, especially poorly water-soluble compounds, high extraction ability and high stabilization ability for some natural products (Choi et al., 2011; Dai et al., 2014; Mamajanov, Engelhart, Bean, \& Hud, 2010). From an environmental and economic perspective, NADES also offer many striking advantages including biodegradability, sustainability, low costs and simple preparation. All these properties make them of interest for applications in health-related areas such as pharmaceuticals, foods, and cosmetics. They have already been

\footnotetext{
* Corresponding author. Fax: +31 715274511.

E-mail address: y.choi@chem.leidenuniv.nl (Y.H. Choi).
}

used to dissolve DNA (Choi et al., 2011; Dai et al., 2013a; Mamajanov et al., 2010), as media for enzyme reactions (Choi et al., 2011; Durand et al., 2013), and biotransformations (Gutiérrez, Ferrer, Yuste, Rojo, \& del Monte, 2010; Zhao, Baker, \& Holmes, 2011) and extraction of phenolics (Dai, Witkamp, Verpoorte, \& Choi, 2013b; Gu et al., 2014; Paiva et al., 2014), processing biomass (Francisco, van den Bruinhorst, \& Kroon, 2012; Xia, Baker, Li, Ravula, \& Zhao, 2014) and stabilization of natural pigment (Dai et al., 2014).

These solvents, however, share some of the limitations observed in conventional synthetic ILs and DES. Their high viscosity (typically $200-500 \mathrm{~mm}^{2} \mathrm{~s}^{-1}$ at $40{ }^{\circ} \mathrm{C}$ ) (Dai et al., 2013a) is the most obvious issue, which leads to some practical problems, including time-consuming solvent transfer operations and slow mass transfer in dissolutions or extractions. In the case of ILs, this is generally partly overcome by applying external physical forces, such as microwave and stirring at high temperature, thus accelerating the dissolving process (Abe, Fukaya, \& Ohno, 2010; Swatloski, Spear, Holbrey, \& Rogers, 2002). Another way to get around this problem is diluting the IL with water, which has proved to be an efficient way to decrease their viscosity and to modulate functional solvents for enzyme reaction (Kohno \& Ohno, 2012). This could also be useful to design appropriate NADES for specific applications, another of the important issues to be solved.

There is an aspect of the effect of water on NADES that adds additional interest to the understanding of the mechanisms involved in the interaction between water and NADES. Water is 
the most abundant liquid on earth and it plays an important role in biological systems. NADES could play a major role as a third type of solvent in cells and living organisms, besides water and lipids. NADES might be involved in the biosynthesis, storage and transport of various poorly water-soluble compounds (Choi et al., 2011; Dai et al., 2013a). From a biological viewpoint, given the coexistence of the two liquids (NADES and water) in organisms, it is of great importance to learn more about how water affects NADES in order to further show their applications. Two explorations on adjusting the behavior of protein in DES (urea-choline chloride) have been done as applications (Esquembre et al., 2013; Nardecchia et al., 2012). Thus, we investigated NADES and water mixtures in terms of supermolecular structures, physicochemical properties, and solubilizing capacity for two poorly water-soluble compounds to demonstrate the role of water addition in developing tailor-made NADES for specific applications. This study provides the basis for a deeper understanding of the chemical structures and physical properties of diluted NADES that can modulate NADES in a controllable way for health-related applications.

\section{Materials and methods}

\subsection{Chemicals, material and reagents}

All the chemicals for the preparation of NADES and quercetin were purchased from Sigma (St. Louis, MO, USA). Water was of deionized water. Deuterium oxide was obtained from CortecNet (Voisins-Le-Bretonneux, France.) Carthamin (purity >95\%) and quercetin (purity $>98 \%$ ) were previously isolated in our laboratory.

\subsection{Solvent preparation}

Natural deep eutectic solvents including proline-malic acid (PM) and 1,2-propanediol-choline chloride-water (molar ratio, $1: 1, P C)$, were prepared by heating with stirring at $70{ }^{\circ} \mathrm{C}$ till a clear liquid was formed. Other NADES including malic acid-choline chloride-water (molar ratio, 1:1:2, MCH), proline-malic acid-water (molar ratio, 1:1:3; PMH), 1,2-propanediol-choline chloride-water (molar ratio, 1:1:1, PCH), lactic acid-glucose-water (molar ratio, $5: 1: 3, \mathrm{LGH}$ ); sucrose-choline chloride-water (molar ratio, 1:4:4, $\mathrm{SuCH}$ ) and other NADES listed in Table 1 were prepared according to our previously reported method: the mixture of two components with certain amount of water was placed in a bottle with a cap and heated in a water bath at $50{ }^{\circ} \mathrm{C}$ with agitation till a clear liquid was formed (Choi et al., 2011; Dai et al., 2013a). 1,2-Propanediol-choline chloride-water (molar ratio, 1:1:1, PCH)

Table 1

The conductivity $\left(\mathrm{mS} \mathrm{cm}^{-1}\right)$ of NADES at room temperature $\left(27^{\circ} \mathrm{C}\right)$.

\begin{tabular}{ll}
\hline Compositions (molar ratio)(abbreviation) & Conductivity \\
\hline Fructose:glucose:sucrose:water $(1: 1: 1: 11)(\mathrm{FGSH})$ & 0.0012 \\
Lactic acid:glucose:water $(5: 1: 3)(\mathrm{LGH})$ & 0.114 \\
Malic acid: $\beta$-alanine:water $(1: 1: 3)(\mathrm{MAH})$ & 0.551 \\
Proline:malic acid:water $(1: 1: 3)(\mathrm{PMH})$ & 1.06 \\
Fructose:choline chloride:water $(2: 5: 5)(\mathrm{FCH})$ & 3.1 \\
Xylose:choline chloride:water $(1: 2: 2)(\mathrm{XCH})$ & 3.15 \\
Sucrose:choline chloride:water $(1: 4: 4)(\mathrm{SuCH})$ & 3.39 \\
Malic acid:choline chloride:water(1:1:2)(MCH) & 5.91 \\
Lactic acid:choline chloride:water $(1: 1)(\mathrm{LC})$ & 6.76 \\
Sorbitol:choline chloride:water $(2: 5: 6)(\mathrm{SoCH})$ & 6.77 \\
Glucose:choline chloride:water $(2: 5: 5)(\mathrm{GCH})$ & 6.81 \\
Xylitol:choline chloride:water $(1: 2: 3)(\mathrm{XoCH})$ & 13.55 \\
1,2-Propanediol:choline chloride:water $(1: 1: 1)(\mathrm{PCH})$ & 12.09 \\
Glycerol:choline chloride:water $(2: 1: 1)(\mathrm{GlyCH})$ & 13.78 \\
Water & 0.00208 \\
Methanol & 0.00424 \\
\hline
\end{tabular}

were diluted with different percentage $(v)$ of deuterium oxide one day before the ${ }^{1} \mathrm{H}$ NMR measurement. For physical properties tests, natural deep eutectic solvents were diluted with deionized water.

\subsection{FT-IR and ${ }^{1} H$ NMR analysis}

The ${ }^{1} \mathrm{H}$ NMR spectra of the diluted $\mathrm{PCH}$ with deuterium oxide were recorded at $40{ }^{\circ} \mathrm{C}$ on a $500 \mathrm{MHz}$ Bruker DMX-500 spectrometer (Bruker, Karlsruhe, Germany). A total of 32,768 data points were recorded covering a spectral window of $9615 \mathrm{~Hz} ; 128$ scans of a standard one-pulse sequence with $90^{\circ}$ flip angle for excitation and presaturation during $2.0 \mathrm{~s}$ relaxation delay. An exponential window function with a line-broadening factor of $0.3 \mathrm{~Hz}$ was applied prior to Fourier transformation. The resulting spectra were manually phased and baseline corrected. FT-IR spectra over the range from 4000 to $300 \mathrm{~cm}^{-1}$ were collected at room temperature $\left(25^{\circ} \mathrm{C}\right)$ using a Bruker FT-IR spectrometer.

\subsection{Physicochemical properties tests}

Viscosity test was performed using a viscometer 16983, type Utube reverse (P.M. Tamson B.V. Zoetermeer, The Netherlands) in a thermostatic bath TVB 445 (Labovisco B.V., The Netherlands) at $40^{\circ} \mathrm{C}$. Conductivity test was recorded at ambient temperature $\left(27^{\circ} \mathrm{C}\right)$ on a $756 \mathrm{KF}$ coulometer (Metrohm) equipped with 728 Stirrer and $756 \mathrm{KF}$ coulometer keyboard). Density test was performed using a density meter (DMA 5000) at $40^{\circ} \mathrm{C}$. Water activity test was performed in a Labmaster water activity equipment (Novasina, Switzerland) at $40^{\circ} \mathrm{C}$. Polarity testing was done with Nile red (NR) as a solvatochromatic probe to estimate polarity of NADES in the form of molar transition energy $\left(E_{\mathrm{NR}}\right)$. The dye was dissolved in NADES in the concentration of $10^{-5}-10^{-4} \mathrm{M}$. The absorption spectra of dyes $\left(\lambda_{\max }\right)$ was determined with a UV/Vis spectrophotometer and calculated in the following formula to obtain $E_{\mathrm{NR}}: E_{\mathrm{NR}}\left(\mathrm{kcal} \mathrm{moL}^{-1}\right)=h c N A / \lambda_{\max }=28,591 / \lambda_{\max }$ (Ogihara, Aoyama, \& Ohno, 2004).

\subsection{Solubility tests}

Solubility tests were carried out by saturating NADES with an excess of the tested compound in a bottle with a cap and stirring at $40^{\circ} \mathrm{C}$ for $2 \mathrm{~h}$. The resulted liquids containing un-dissolved solid compounds were centrifuged and the supernatant was transferred to a $1.5 \mathrm{~mL}$-microtube, diluted with water, and vortexed till a homogeneous solution. The diluted solutions were analyzed by UV spectrometer at wavelength of $370 \mathrm{~nm}$ for quercetin and $517 \mathrm{~nm}$ for carthamin. All the solubility tests were performed in triplicate.

\section{Results and discussion}

\subsection{The supermolecular structure of NADES}

The supermolecular structure of NADES were investigated with ${ }^{1} \mathrm{H}-{ }^{1} \mathrm{H}$-nuclear overhauser enhancements (NOESY) spectroscopy (Dai et al., 2013a), and also further elucidated with FT-IR (Fig. 1) to explore the type of interactions, the atoms involved in the interactions and even the possible ratios of the two components. The FT-IR spectra of solid malic acid, proline and their eutectic mixture malic acid, proline (molar ratio, 1:1) were recorded at room temperature. Compared with single compounds, an upward shift of the stretching vibration band $v_{\mathrm{CO}}$ in malic acid from 1683 to $1717 \mathrm{~cm}^{-1}$ and a downward shift of $v_{\mathrm{OH}}$ to ca. 2450 and $1920 \mathrm{~cm}^{-1}$ were observed (Fig. 1a), indicative of the appearance 
<smiles>O=C(O)[C@@H]1CCC[C@@H]1[AlH2]</smiles>

malic acid proline

a
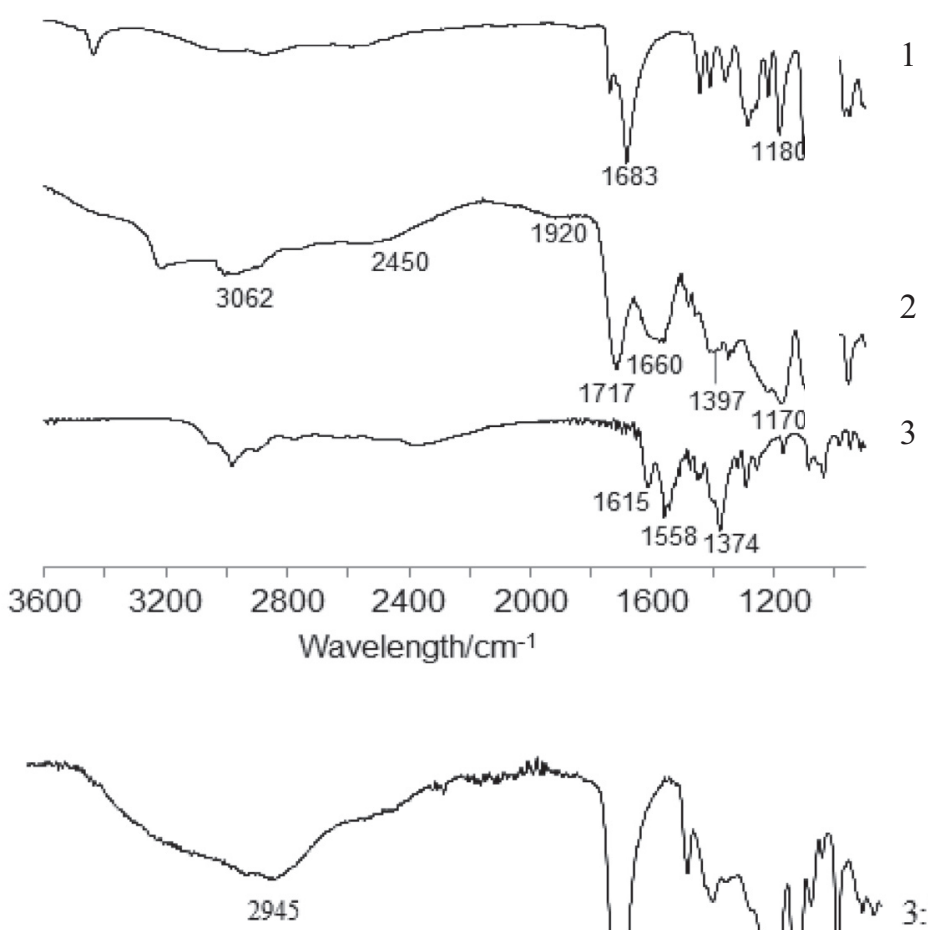

b

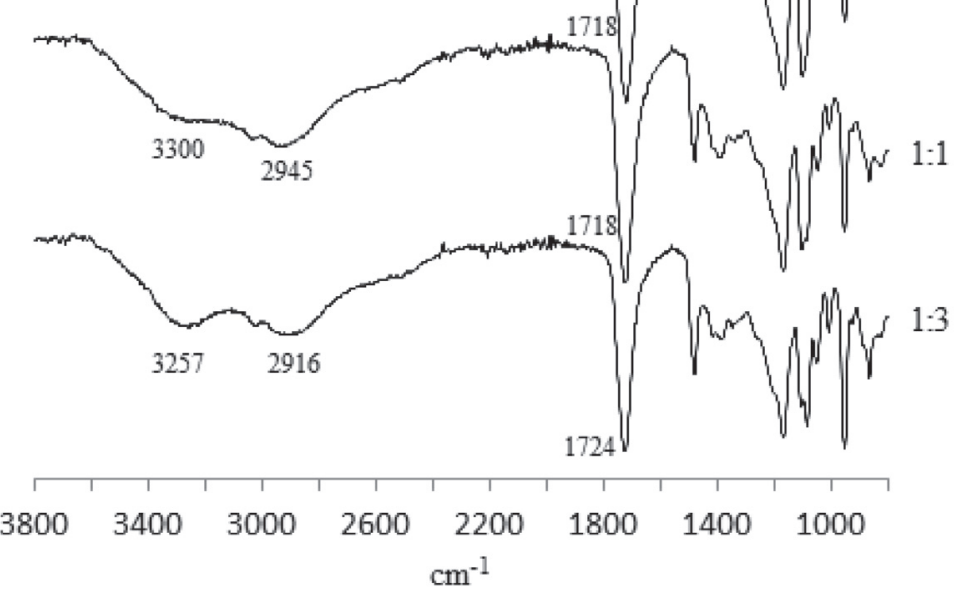

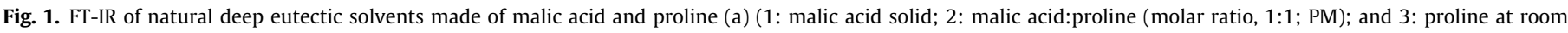
temperature) and (b) eutectic mixture of malic acid:proline with different molar ratio $(3: 1 ; 1: 1 ; 1: 3)$.

of a hydrogen bond $(\mathrm{O}=\mathrm{C}-\mathrm{O}-\mathrm{H} \cdots \mathrm{N})$ involving malic acid and proline in PM (Barańska, Kuduk-Jaworska, Szostak, \& Romaniewska, 2003; Xu et al., 2005). In PM, the deformation vibration band of $\delta_{\mathrm{CH} 2}$ in malic acid increased in intensity and shifted from 1180 to $1170 \mathrm{~cm}^{-1}$, and in proline the $\delta_{\mathrm{COO}-\mathrm{H}}$ shifted from 1374 to $1397 \mathrm{~cm}^{-1}$, and the $\delta_{\mathrm{NH}}$ shifted from 1558 to $1660 \mathrm{~cm}^{-1}$. This suggests that both malic acid and proline have different conformations in the PM to form hydrogen bond (Mary, Ushakumari, Harikumar, Varghese, \& Panicker, 2009).

Furthermore, the IR spectra of different molar ratios $(1: 3 ; 1: 1$; $3: 1)$ in PM showed that the band of $v_{\mathrm{C}=\mathrm{O}}$ at $1708 \mathrm{~cm}^{-1}$ shifts up to $1716 \mathrm{~cm}^{-1}$ and the broad band of $v_{\mathrm{OH}}$ in carboxyl group shifts from 2972 to $2980 \mathrm{~cm}^{-1}$, indicating that more $\mathrm{H}$-bonds between proline and malic acid are formed when the ratio of proline-malic acid increased from 1:3 to 1:1 (Fig. 1b). When the amount of proline-malic acid increased to $3: 1$, there is no shift of the $v_{\mathrm{CO}}$ and $v_{\mathrm{OH}}$ in carboxyl group. This phenomenon suggests that $1: 1$ is a proper ratio for malic acid and proline to form a eutectic mixture. Similar hydrogen bonds were also detected in the 1,2-propanediol-choline chloride eutectic mixture (PC) (Fig. S-1), including $\mathrm{H}$-bonds between hydroxyl groups in 1,2-propanediol and chloride, and a hydrogen bond with $-\mathrm{CH}_{2}-\mathrm{OH}$ in choline chloride. All structure 
information confirms and extends our reported 2D NMR results of PMH and PCH (Dai et al., 2013a). Clearly, there is an extensive hydrogen-bonding network between the components of NADES; thus, NADES are supermolecules with hydrogen bond network.

\subsection{The supermolecular structure of NADES after water dilution}

To explore the supermolecular structure of NADES after dilution with water, the eutectic mixture 1,2-propanediol-choline chloride-water $(1: 1: 1)(\mathrm{PCH})$ was diluted with deuterium oxide and the diluted PCH was investigated with NMR (Fig. S-2). A continuous downfield shift of all signals from the two components except the peaks of methyl and methylene groups vicinal to the nitrogen atom was observed, and the relative number of detected protons on the hydroxyl groups (relative to the protons on the methyl group) decreased during dilution (Table S-1). These observations provide evidence of the progressive rupture of the hydrogen bonds in PCH during dilution, a similar phenomenon was also observed in urea-choline chloride mixture by Gutiérrez, Ferrer, Mateo, and del Monte (2009). The dilution from 25\% to 50\%, caused all the signals of the hydroxyl groups to disappear, but no shift of the water signal was observed with a further dilution to $75 \% \mathrm{D}_{2} \mathrm{O}$, indicating the complete rupture of the hydrogen bonds between 1,2-propanediol and choline chloride with $50 \% \mathrm{D}_{2} \mathrm{O}$. These results show that the supermolecular complex structures of $\mathrm{PCH}$ are preserved while the content of water is below $50 \%$ water, but further dilution produces a solution of the free forms of the individual components in water. These gradual changes in the structure of NADES during dilution may affect their physicochemical properties and also their applications.

\subsection{The physicochemical properties of NADES after water dilution}

\subsubsection{Viscosity}

The viscosity of $\mathrm{PCH}$ diluted with different percentage of water was measured at room temperature. The viscosity decreased significantly as the water content increased following a function $y=0.88 x^{-1.524}\left(r^{2}=0.99\right)(y$ : viscosity; $x$ : water weight percentage in PCH) (Fig. 2), similar as the case of glucose-choline chloride-water (GCH) (Dai et al., 2013a). The result shows that the viscosity of NADES is greatly affected by the water content. The high viscosity of NADES is often attributed to the presence of extensive hydrogen-bonding interactions between the components. Dilution with water leads to a large decrease in the viscosity of NADES as a result of the gradually weakened hydrogen-bonding interactions between the components, as revealed in Fig. S-2. After adding $25 \%$ water to glucose-choline-water (GCH) and $\mathrm{PCH}$, their viscosity decreased from 397 to $7.2 \mathrm{~mm}^{2} \mathrm{~s}^{-1}$ (Dai et al., 2013a), and from 33 to $6.1 \mathrm{~mm}^{2} \mathrm{~s}^{-1}$, respectively, which is near the range of water $\left(0.7 \mathrm{~mm}^{2} \mathrm{~s}^{-1}\right)$. What's more, NADES still possess the supermolecular characteristics with less than $50 \%$ water dilution as mentioned above. Thus, dilution with water not only can effectively reduce the viscosity of NADES but also keep their structure, which may

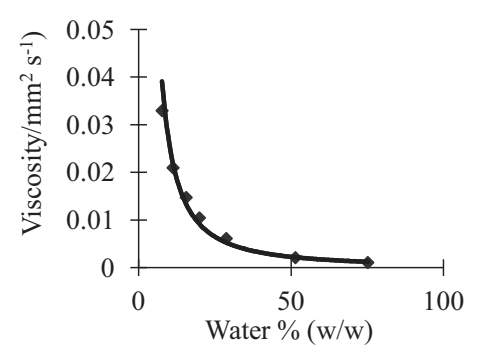

Fig. 2. The relationship curve between the viscosity and water percentage of 1,2propanediol-choline chloride $(1: 1 ; \mathrm{PC})$. facilitate their different applications. These results reveal the role of water as a diluting agent with respect to its considerable efficiency in adjusting the viscosity of NADES.

\subsubsection{Conductivity}

The conductivity of 16 typical NADES and five NADES diluted with water was investigated at room temperature. Polyalcoholcholine had the highest conductivity $\left(\mathrm{GlyCH}, 13.78 \mathrm{mS} \mathrm{cm}^{-1}\right)$ while sugar-sugar (FGSH, $0.001 \mathrm{mS} \mathrm{cm}^{-1}$ ) had the lowest value, similar to water $\left(0.002 \mathrm{mS} \mathrm{cm}^{-1}\right)$ and methanol $\left(0.004 \mathrm{mS} \mathrm{cm}^{-1}\right)$ (Table 1 ). The conductivity of NADES decreases in the following sequence: base-polyalcohol $>$ base-organic acid $\approx$ base-sugar $>$ organic acid-amino acid (non-polar) $>$ organic acid-sugar $>$ sugarsugar. NADES with high and low conductivity $(\mathrm{PCH}, \mathrm{GCH}$, sucrose-choline chloride-water $(\mathrm{SuCH}), \mathrm{LGH}$ and $\mathrm{PMH}$ ) were selected to investigate how the conductivity is affected by water. The conductivity of five NADES diluted with different percentage of water showed that conductivity firstly increased with the increasing water content, and then decreased after reaching a peak value of around 10-100 times higher than that of pure NADES (Fig. 3). GCH, PCH, sucrose-choline chloride-water ( $\mathrm{SuCH}$ ) and $\mathrm{PMH}$ had the highest conductivity with $60 \%$ of water dilution and LGH reached the highest value with $80 \%$ water dilution. Therefore, the conductivity of NADES thus increases as a result of the decreased viscosity by dilution with certain amount of water and the conductivity of NADES can be tailored by changing the water content.

Our results suggest that the conductivity of NADES is correlated with their composition and viscosity. Comparing NADES with one same component, $\underline{\mathrm{GCH}}\left(6.81 \mathrm{mS} \mathrm{cm}^{-1}\right)$ was observed to have a much higher conductivity than $\mathrm{LGH}\left(0.11 \mathrm{mS} \mathrm{cm}^{-1}\right)$, and $\underline{\mathrm{MCH}}$ $\left(5.91 \mathrm{mS} \mathrm{cm}^{-1}\right)$ was much more conductive than $\mathrm{PMH}$ $\left(1.06 \mathrm{mS} \mathrm{cm}^{-1}\right)$, which may be due to the ionic form of choline chloride and partial ionization of lactic acid ( $p k a$ 3.86) and proline (pka 1.99). Thus, it may be concluded that NADES with choline chloride have a high conductivity while NADES with organic acid
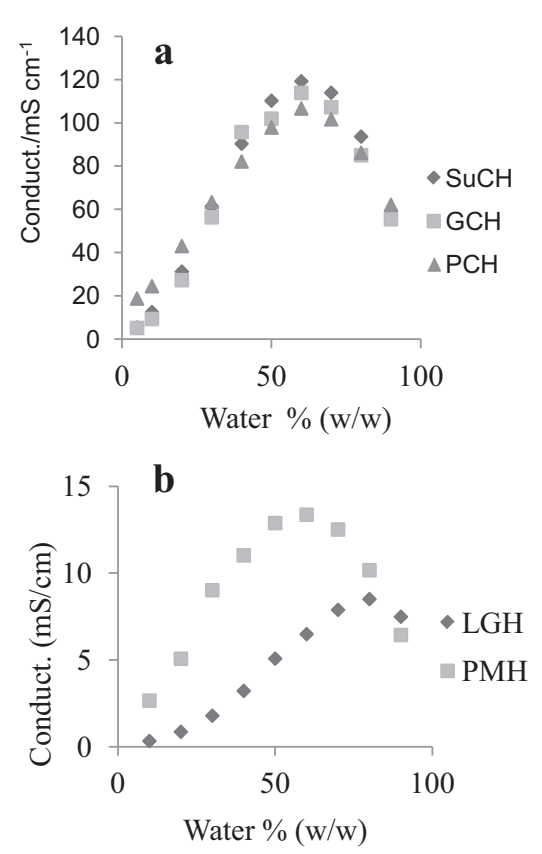

Fig. 3. The relationship curves between the conductivity and water percentage of glucose-choline chloride (molar ratio, 1:2.5; GCH), sucrose-choline chloride (1:4; $\mathrm{SuCH}), 1,2$-propanediol-choline chloride $(1: 1 ; \mathrm{PCH})$, lactic acid:glucose $(5: 1 ; \mathrm{LG})$ and proline:malic acid $(1: 1 ; \mathrm{PMH})$ at room temperature. 
or amino acid have low values. The second important factor is viscosity. NADES made of a polyalcohol and a base such as $\mathrm{PCH}$ are more conductive than those made of a sugar and a base such as $\mathrm{SuCH}$, which might be correlated to the fact that $\mathrm{PCH}$ has much lower viscosity $\left(33 \mathrm{~mm}^{2} \mathrm{~s}^{-1}\right)$ than $\mathrm{SuCH}\left(581 \mathrm{~mm}^{2} \mathrm{~s}^{-1}\right.$ ) (Dai et al., 2013a). Most highly viscous NADES, especially NADES containing a sugar or amino acid $\left(300-600 \mathrm{~mm}^{2} \mathrm{~s}^{-1}\right)$, have a low conductivity (lower than 10) (Dai et al., 2013a).

\subsubsection{Density, water activity, and polarity}

Other physical properties (e.g. density, water activity and polarity) are also affected by the water content. The density, water activity and polarity of some typical NADES diluted with different percentage of water were detected. Results showed that the density of GCH decreased linearly with the increasing water content following a function $y=-0.0024 x+1.224, r^{2}=0.99$ ( $y$ : density; $x$ : water weight percentage in $\mathrm{PCH}$ ) (Fig. 4a). A similar linear relationship was also observed in $\mathrm{SuCH}(y=-0.0025 x+1.2418, r=0.99)$ and PCH $(y=-0.0009 x+1.0873, r=0.99)$. With these formulas, the density of diluted NADES can be calculated with known amount of water dilution.

The water activity of NADES increased gradually with their increasing water content following a function $y=0.354$ $\ln (x)-0.6008$ for GCH ( $y$ : water activity; $x$ : water weight percentage in GCH) (Fig. 4b). Interestingly, above a $50 \%$ dilution with water, there was a linear relationship $(y=0.004 x+0.6016$, $r^{2}=0.99$ ) between the water content and water activity. This change in water activity might be correlated with the above-described effect of a $50 \%$ water dilution on the supermolecular structure of NADES. It implies that the change in the physical properties of NADES may reflect the change in chemical structure during dilution. In addition, NADES covers a wide range of polarity, from more polar than water to a similar polarity to methanol. Water dilution has a great effect on the polarity of NADES resulting in a polarity similar to water itself (Dai et al., 2013a). All this results shows that also the physical properties of NADES can be tailored in a controlled way by adding water.

\subsection{Application of water-diluted NADES in natural products research}

The possibility of adjusting NADES properties quantitatively by adding water allows them to be adapted to different applications
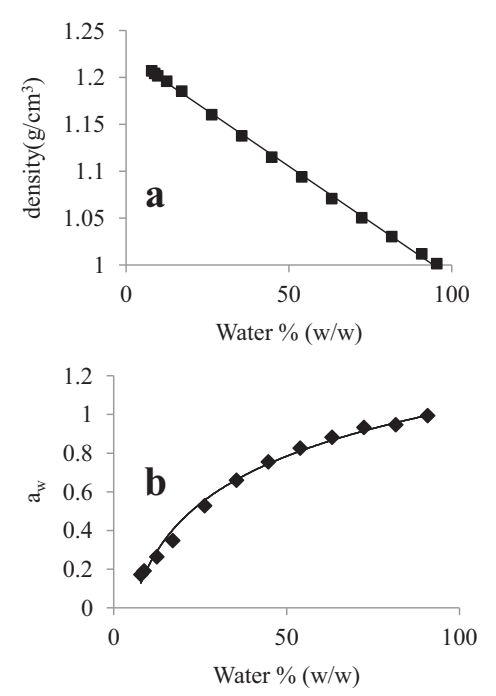

Fig. 4. The relationship curves between (a) the density $\left(\mathrm{g} \mathrm{cm}^{-3}\right)$ and water percentage of 1,2-propanediol-choline chloride (1:1) and (b) between the water activity and water percentage of glucose-choline (2:5). such as enzyme reaction (Choi et al., 2011). Another important application of NADES as the third solvent in organs is their high solubilizing capacity for natural products. The solubility of two poorly water-soluble compounds quercetin and carthamin was investigated in $\mathrm{PCH}$ with different water contents. The results show that solubility of quercetin was decreased with $5 \%$ water in $\mathrm{PCH}$, keeping similar solubility with $10 \%(\mathrm{v} / \mathrm{v})$ water but decreasing drastically upon dilution with $25 \%$ water (v/v) water (Fig. 5). Notably, the case is different for carthamin (a natural dye and antioxidant) (Takahashi et al., 1982; Watanabe, Hasegawa, Yamamoto, Nagai, \& Terabe, 1997), which was most soluble in PCH with $5 \%$ water, probably due to the medium polarity of carthamin. PCH has the lowest polarity of all our tested NADES (Dai et al., 2013a), and the dilution leads to an increase of its polarity and consequently to a value similar to the polarity of water (Dai et al., 2013a). So the solubility of solutes in diluted PCH will change a lot with water dilution. Most interestingly, the solubility of both quercetin and carthamin decrease strongly when the water content increases from $25 \%$ to $50 \%$ in $\mathrm{PCH}$, reaching nearly the same values as in water. These phenomena are coherent with the above-mentioned effect of a $50 \%$ water dilution on the structure of $\mathrm{PCH}$. The decrease of solubility might be correlated with the disappearance of hydrogen bond network with $50 \%$ water dilution.

Our results imply that the changed polarity of diluted NADES have a big effect on the solubilization capacity of NADES. This solubility is also correlated with the polarity of solutes, and nonpolar compounds have the highest solubility in pure NADES while a 5$10 \mathrm{v} \%$ of water dilution allows the highest solubility of medium polar compounds, which is consist with our former study (Dai et al., 2013a). Furthermore, for different NADES, the optimal water content may be also correlated with the viscosity of NADES. For example, carthamin has the highest solubility in $\mathrm{PCH}$ with $5 \%$ water and in $\mathrm{GCH}$ with $10 \%$ water although GCH is more polar than $\mathrm{PCH}$, and this is correlated with the higher viscosity of GCH (Dai et al., 2013a). This points to an extensive hydrogen bonding structure in GCH which results in a high viscosity where there is no space for dissolving various solutes and more water is needed to
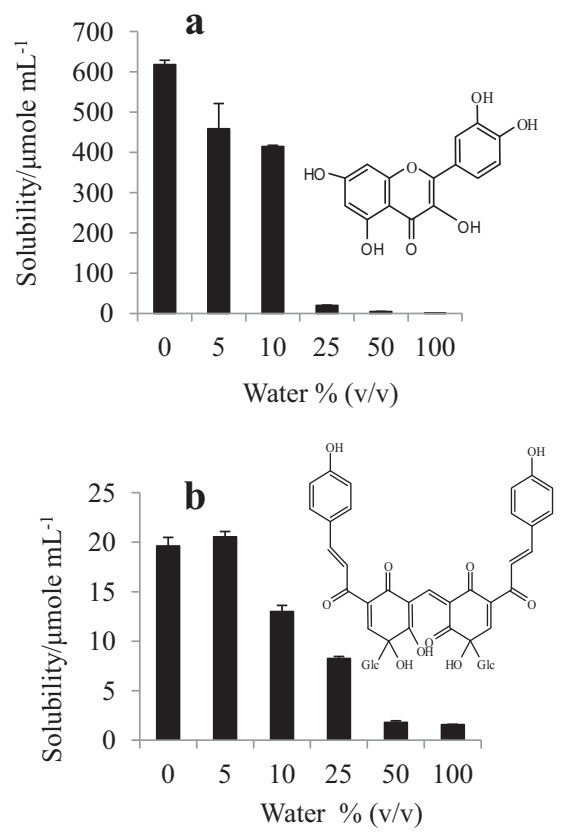

Fig. 5. The solubility of quercetin (a) and carthamin (b) in $\mathrm{PCH}$ diluted with a different percentage of water. The data is expressed in mean $\pm \operatorname{SD}(n=3)$. ${ }^{*} p<0.1$; ${ }^{* *} p<0.01$. 
loosen the bonded structure and dissolve them. So the physicochemical characteristics of NADES with different water contents are useful to predict the solubilization capacity of NADES.

\section{Conclusion}

NADES are supermolecules with hydrogen-bonding interactions between the components. The interactions will be weakened with water dilution and even disappear when the water content is above $50 \%(\mathrm{v} / \mathrm{v})$. The physicochemical properties can be tailored in a controllable way when diluted with water, as is the case of their viscosity that can be decreased almost to that of water with the addition of $25 \%$ water $(v / v)$. The effect of water content on the structure and characteristics of NADES provide a basis to develop tailor-made NADES for various applications, such as the dissolution of compounds, revealing their great potential for diverse uses in health related areas.

\section{Acknowledgments}

This work was supported by NWO ECHO Grant (Project No. 711.012.010) and partially by and a Horizon Valorisation Grant (Project No. 93515516). The authors thank Dr. Erica G. Wilson at Leiden University for language revision. Yuntao Dai thanks the support from PhD program of the China Scholarship Council.

\section{Appendix A. Supplementary data}

Supplementary data associated with this article can be found, in the online version, at http://dx.doi.org/10.1016/j.foodchem.2015. 03.123.

\section{References}

Abe, M., Fukaya, Y., \& Ohno, H. (2010). Extraction of polysaccharides from bran with phosphonate or phosphinate-derived ionic liquids under short mixing time and low temperature. Green Chemistry, 12, 1274-1280.

Barańska, H., Kuduk-Jaworska, J., Szostak, R., \& Romaniewska, A. (2003). Vibrational spectra of racemic and enantiomeric malic acids. Journal of Raman Spectroscopy, $34,68-76$.

Choi, Y. H., Van Spronsen, J., Dai, Y., Verberne, M., Hollmann, F., Arends, I. W. C. E. et al. (2011). Are natural deep eutectic solvents the missing link in understanding cellular metabolism and physiology? Plant Physiology, 156, 1701-1705.

Dai, Y., Spronsen, J. V., Witkamp, G. J., Verpoorte, R., \& Choi, Y. H. (2013a). Natura deep eutectic solvents as new potential media for green technology. Analytica Chimica Acta, 766, 61-68.
Dai, Y., Verpoorte, R., \& Choi, Y. H. (2014). Natural deep eutectic solvents providing enhanced stability of natural colorants from safflower (Carthamus tinctorius). Food Chemistry, 159, 116-121.

Dai, Y., Witkamp, G. J., Verpoorte, R., \& Choi, Y. H. (2013b). Natural deep eutectic solvents as new extraction media for phenolic metabolites in safflower. Analytic Chemistry, 85, 6272-6278.

Durand, E., Lecomte, J., Barea, B., Dubreucq, E., Lortie, R., \& Villeneuve, P. (2013). Evaluation of deep eutectic solvent-water binary mixtures for lipase-catalyzed lipophilization of phenolic acids. Green Chemistry, 15, 2275-2282.

Esquembre, R., Sanz, J. M., Wall, J. G., del Monte, F., Mateo, C. R., \& Ferrer, M. L. (2013). Thermal unfolding and refolding of lysozyme in deep eutectic solvents and their aqueous dilutions. Physical Chemistry Chemical Physics, 15, $11248-11256$

Francisco, M., van den Bruinhorst, A., \& Kroon, M. C. (2012). New natural and renewable low transition temperature mixtures (LTTMs): Screening as solvents for lignocellulosic biomass processing. Green Chemistry, 14, 2153-2157.

Gu, T., Zhang, M., Tan, T., Chen, J., Li, Z., Zhang, Q., et al. (2014). Deep eutectic solvents as novel extraction media for phenolic compounds from model oil. Chemical Communications, 50, 11749-11752.

Gutiérrez, M. C., Ferrer, M. L., Mateo, C. R., \& del Monte, F. (2009). Freeze-drying of aqueous solutions of deep eutectic solvents: A suitable approach to deep eutectic suspensions of self-assembled structures, Langmuir, 25, 5509-5515.

Gutiérrez, M. C., Ferrer, M. L., Yuste, L., Rojo, F., \& del Monte, F. (2010). Bacteria incorporation in deep-eutectic solvents through freeze-drying. Angewandte Chemie International Edition, 49, 2158-2162.

Kohno, Y., \& Ohno, H. (2012). Ionic liquid/water mixtures: From hostility to conciliation. Chemical Communications, 48, 7119-7130.

Mamajanov, I., Engelhart, A., Bean, H., \& Hud, N. (2010). DNA and RNA in anhydrous media: Duplex, triplex, and G-quadruplex secondary structures in a deep eutectic solvent. Angewandte Chemie International Edition, 49, 6310-6314.

Mary, Y. S., Ushakumari, L., Harikumar, B., Varghese, H. T., \& Panicker, C. Y. (2009). FT-IR, FT-Raman and SERS spectra of L-proline. Journal of the Iranian Chemical Society, 6, 138-144.

Nardecchia, S., Gutiérrez, M. C., Ferrer, M. L., Alonso, M., López, I. M., RodríguezCabello, J. C., et al. (2012). Phase behavior of elastin-like synthetic recombinamers in deep eutectic solvents. Biomacromolecules, 13, 2029-2036.

Ogihara, W., Aoyama, T., \& Ohno, H. (2004). Polarity measurement for ionic liquids containing dissociable protons. Chemistry Letters, 33, 1414-1415.

Paiva, A., Craveiro, R., Aroso, I., Martins, M., Reis, R. L., \& Duarte, A. R. C. (2014). Natural deep eutectic solvents - Solvents for the 21st century. ACS Sustainable Chemistry \& Engineering, 2, 1063-1071.

Swatloski, R. P., Spear, S. K., Holbrey, J. D., \& Rogers, R. D. (2002). Dissolution of cellose with ionic liquids. Journal of American Chemical Society, 124, 4974-4975.

Takahashi, Y., Miyasaka, N., Tasaka, S., Miura, I., Urano, S., Ikura, M., et al. (1982). Constitution of two coloring matters in the flower petals of Carthamus tinctorius L. Tetrahedron Letters, 23, 5163-5166.

Watanabe, T., Hasegawa, N., Yamamoto, A., Nagai, S., \& Terabe, S. (1997). Separation and determination of yellow and red safflower pigments in food by capillary electrophoresis. Bioscience Biotechnology and Biochemistry, 61, 1179-1183.

Xia, S., Baker, G. A., Li, H., Ravula, S., \& Zhao, H. (2014). Aqueous ionic liquids and deep eutectic solvents for cellulosic biomass pretreatment and saccharification. RSC Advances, 4, 10586-10596.

Xu, J. W., Toh, C. L., Liu, X. M., Wang, S. F., He, C. B., \& Lu, X. H. (2005). Synthesis and self-assembly of donor-spacer-acceptor molecules. Liquid crystals formed by single-component "complexes" via intermolecular hydrogen-bonding interaction. Macromolecules, 38, 1684-1690.

Zhao, H., Baker, G. A., \& Holmes, S. (2011). New eutectic ionic liquids for lipase activation and enzymatic preparation of biodiesel. Organic \& Biomolecular Chemistry, 9, 1908-1916. 